\title{
Perfil do estado de saúde de mulheres climatéricas
}

\section{Health status profile of climacteric women}

Roberta DS Santos ${ }^{1}$; Flávia GD Carvalho²; Tatiana P Lima3; Riana L Viegas; Angélica Faria ${ }^{4}$; Vivian MM Suen; Anderson M Navarro'; Renata lannetta ${ }^{1}$; Carla B Nonino ${ }^{6}$; Julio S Marchini; Odilon lannetta ${ }^{7}$

\begin{abstract}
RESUMO
Modelo do estudo: Estudo retrospectivo com análise de dados de prontuário.

Objetivo: O presente estudo teve por objetivo verificar a freqüência de obesidade, diabetes mellitus, hipertensão arterial e dislipidemia em um grupo de mulheres climatéricas.

Metodologia: Estudo das primeiras pacientes atendidas no Ambulatório do Climatério (ACLI) do Departamento de Ginecologia e Obstetrícia da Faculdade de Medicina de Ribeirão Preto -USP, no período de 1983 a 2007. De fevereiro a abril de 2008 foram coletados dados de peso, estatura, Índice de Massa Corporal (IMC), grupo biológico, diagnóstico de hipertensão, diabetes e dislipidemia.

Resultados: De 1983 a 2007, 400 pacientes tiveram seguimento no Ambulatório (ACLI), e 272 apresentavam seus prontuários com os dados do presente estudo devidamente registrados. Dessas 272 pacientes, foram selecionadas 628 consultas, sendo que, portanto, em média, cada mulher teve três retornos. Estas mulheres tinham idade mínima de 29 e máxima de 80 anos, com IMC mediano acima de $25 \mathrm{~kg} / \mathrm{m}^{2}$. O diagnóstico de diabetes, hipertensão e dislipidemia foi detectado em, respectivamente: $32 \%, 68 \%$ e $54 \%$ dos casos.

Conclusão: Mulheres climatéricas atendidas em um hospital de nível de atendimento terciário apresentaram um aumento do IMC e da prevalência de doenças crônicas não transmissíveis com o passar do tempo, o que faz urgir um olhar mais atento dos profissionais de saúde a esse grupo populacional.
\end{abstract}

Palavras-chave: Climatério. Menopausa. Hipertensão. Diabetes Mellitus. Dislipidemias. Obesidade.

\section{Introdução}

A expectativa de vida da mulher brasileira situa-se, atualmente, em torno dos 70 anos, com a idade da menopausa permanecendo próxima dos 50 anos. Entende-se, portanto, que a mulher viva um terço ou mais da vida após a menopausa. ${ }^{1}$
O climatério, que ocorre entre 39-65 anos, representa a transição entre as fases reprodutivas e não reprodutivas, sendo que esta fase pode ser subdividida em grupos biológicos. No serviço de Climatério do Hospital das Clínicas de Ribeirão Preto (HCFMRP/ USP), é utilizada rotineiramente, por toda a equipe, a divisão das mulheres atendidas em sete grupos bioló-
1. Doutoranda pela USP - Campus Ribeirão Preto.

2. Doutoranda pela UNESP- Campus Araraquara.

3. Mestranda pela USP- Campus Ribeirão Preto.

4. Nutricionista.

5. Médica associada ao Departamento de Clínica Médica, USPCampus Ribeirão Preto.

6. Docente. Curso de Nutrição e Metabolismo, USP- Campus Ribeirão Preto.

7. Docente. Curso de Ciências Médicas, USP- Campus Ribeirão Preto.
Correspondência:

Roberta DS Santos.

Departamento de Clínica Médica - HCRP Avenida dos Bandeirantes, $\mathrm{n}^{\circ} 3900$, Bairro Monte Alegre, 14049-900. Ribeirão Preto/SP robertinha_nutriusp@yahoo.com.br

Artigo recebido em 16/04/2012 Aprovado para publicação em 20/07/2012 
gicos, de acordo com a regularidade e presença ou ausência do ciclo menstrual: A- Ciclos menstruais regulares, B- Ciclos menstruais irregulares, C1- Menopausa há um ano, C2- Menopausa há dois anos, C3Menopausa há três anos, C4- Menopausa há quatro anos, C5- Menopausa há cinco anos ou mais. ${ }^{2}$ Esta fase caracteriza-se pela diminuição da função reprodutiva feminina, com conseqüências para o equilíbrio metabólico e endócrino. ${ }^{1}$

Vários agravos à saúde constatados no climatério relacionam-se também, direta ou indiretamente, com a ingestão inadequada de alimentos, quer seja o excesso ou a deficiência por longos períodos. ${ }^{3}$ De acordo com Kimura et al (2002), a ausência de estrogênio pode estar relacionada com a diminuição de receptores de leptina no hipotálamo, o que compromete os mecanismos de controle de fome e saciedade, resultando em uma diminuição da saciedade e consequentemente maior ingestão de alimentos e maior ganho de massa corpórea. ${ }^{4}$ Essa desregulação no consumo de alimentos favorece o ganho de peso e gordura corporais bem como alterações desfavoráveis no perfil lipídico, o qual por sua vez, constituem fatores de risco para inúmeras doenças crônicas não-transmissíveis $^{4}$, tais como as cardiovasculares, diabetes mellitus tipo II, obesidade, dislipidemias, osteoporose, câncer de cólon e de mama. ${ }^{5,6}$

No caso específico da mulher climatérica, os excessos nutricionais relacionam-se com o maior consumo de alimentos energéticos, principalmente provenientes de gorduras saturadas. A ingestão de alimentos com elevada porcentagem de gordura é considerada determinante primária de obesidade. ${ }^{3}$

O ganho de peso parece não estar linearmente relacionado ao envelhecimento, mas aumenta especificamente nos anos pós-menopausa. No entanto, não está claro na literatura, se o ganho ponderal está relacionado ao processo de envelhecimento, às flutuações hormonais inerentes ao climatério ${ }^{7,8}$, ou à uma combinação de ambos os fatos ${ }^{9}$. Segundo Monteiro et al. $(2004)^{10}, 60 \%$ das mulheres na idade da menopausa apresentam um rápido ganho de peso entre 2,5 a 5,0 $\mathrm{kg}$, enquanto que, segundo Eshtiaghi et al. (2010) ${ }^{11}$, mulheres na meia-idade apresentam um ganho de peso de $0,5 \mathrm{~kg} /$ ano após a menopausa, e para Lovejoy $(1998)^{7}$, a maior parte das mulheres ganha entre 0,9 a 2,3 kg durante a transição menopausal, podendo corresponder a $20 \%$ da gordura corporal total. ${ }^{12}$

Diante do aumento da incidência de doenças crônico-degenerativas que podem se instalar no perí- odo do climatério, o presente estudo teve por objetivo verificar a evolução do peso bem como da frequência de obesidade, diabetes mellitus, hipertensão arterial e dislipidemias, em mulheres climatéricas atendidas no Ambulatório do Climatério (ACLI) do Departamento de Ginecologia e Obstetrícia da Faculdade de Medicina de Ribeirão Preto -USP, no período de 1983 a 2007.

\section{Materiais e métodos}

Foi realizado um estudo retrospectivo baseado em coleta de dados de prontuários médicos de mulheres atendidas no Ambulatório Multidisciplinar de Climatério do Hospital das Clínicas da Faculdade de Medicina de Ribeirão Preto-USP (HCFMRP-USP), no período de 1983 a 2007. O estudo foi previamente aprovado pelo Comitê de Ética em Pesquisa do HCFMRP-USP. Os prontuários foram analisados no período de fevereiro a abril de 2008.

Para o estudo, foram selecionadas as primeiras pacientes que foram atendidas no Ambulatório, por volta da década de 80 , e que tiveram seguimento até a primeira década de 2000. Sendo assim, as pacientes do ambulatório atendidas na década de 80 que perderam seguimento até a primeira década de 2000 , não foram inclusas nas análises. Além disso, foram considerados como fatores de exclusão pacientes portadoras de neoplasias e disfunções na tireoide e prontuários incompletos (Figura 1).

Dos prontuários, foram coletados os seguintes dados: idade, peso, altura, Índice de Massa Corporal (IMC), grupo biológico, diagnóstico de Diabetes Mellitus, Hipertensão Arterial e Dislipidemia. O IMC foi classificado segundo a Organização Mundial de Saúde (2012). ${ }^{13}$ Para a definição de grupo biológico, considerou-se a regularidade e presença ou ausência do ciclo menstrual, sendo agrupadas em sete grupos biológicos a seguir: A- Ciclos menstruais regulares, BCiclos menstruais irregulares, C1- Menopausa há um ano, C2- Menopausa há dois anos, C3- Menopausa há três anos, C4- Menopausa há quatro anos, C5- Menopausa há cinco anos ou mais. ${ }^{2}$

\section{Análise dos resultados}

Os dados foram organizados e apresentados segundo grupo biológico e período de estudo. Foi realizada análise estatística descritiva, utilizando o programa EpiData Analysis ${ }^{\circledR}$ sendo apresentados os valores mínimo, mediana, máximo e frequência das observações. 


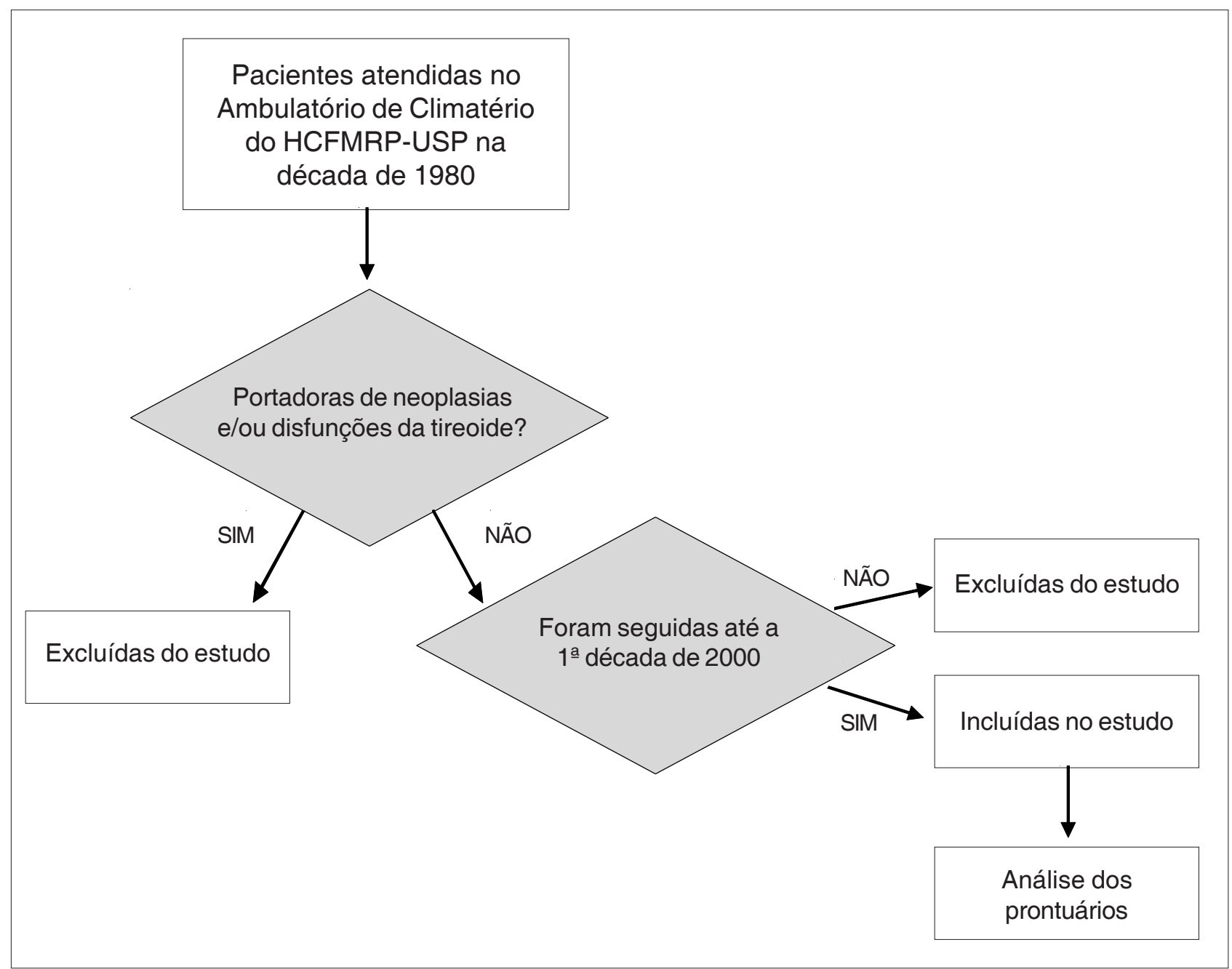

Figura 1: Fluxograma de seleção de pacientes para a pesquisa.

\section{Resultados}

No período de 1983 a 2007, 400 pacientes tiveram seguimento no Ambulatório (ACLI), e 272 apresentavam seus prontuários com os dados do presente estudo devidamente registrados. Dessas 272 pacientes, foram selecionadas 628 consultas, sendo que, portanto, em média, cada mulher teve 3 retornos.

A Tabela 1 representa o número de atendimentos realizados no Ambulatório do Climatério, entre 1983 a 2007. Pode-se observar que o número de atendimentos declinou bem como o perfil das mulheres atendidas alterou-se com o passar do tempo. Um número maior de mulheres do grupo biológico $\mathrm{C} 5$ foram atendidas no período de 2000 a 2007, em detrimento das mulheres mais jovens.

Na Tabela 2 estão representados o número de pacientes pertencentes aos grupos biológicos, a me- diana, valores máximo e mínimo da idade e Índice de Massa Corporal (IMC). Observa-se que há mais pacientes pertencentes ao grupo biológico $\mathrm{C} 5$, o que corrobora a Tabela 1. A idade mínima foi de 29 anos e a máxima, 80 anos.

A frequência do excesso de peso foi alta independente do grupo biológico com valores de IMC acima de $25 \mathrm{~kg} / \mathrm{m}^{2}$. Estes valores foram ainda maiores para as mulheres em grupos biológicos tardios, apresentando valores de IMC máximos de $48 \mathrm{~kg} / \mathrm{m}^{2}$.

Já a Tabela 3 ilustra a prevalência de subnutrição, eutrofia, sobrepeso e obesidade no período do estudo (1983 a 2007). Durante este período houve uma diminuição da prevalência de subnutrição (2,6 para $0 \%)$, eutrofia (de 36,8 para 22,0\%) e sobrepeso (de 34,9 para $28,8 \%$ ) e aumento da prevalência de obesidade (de 25,7 para 49,2\%). 
Tabela 1

Distribuição percentual da freqüência de atendimentos segundo grupo biológico e período de estudo

\begin{tabular}{|c|c|c|c|c|c|c|c|c|c|c|c|c|c|c|c|c|}
\hline \multirow[b]{3}{*}{ Período } & \multicolumn{16}{|c|}{ Grupos Biológicos } \\
\hline & \multicolumn{2}{|c|}{ A } & \multicolumn{2}{|c|}{ B } & \multicolumn{2}{|c|}{$\mathrm{Cl}$} & \multicolumn{2}{|c|}{$\mathrm{C} 2$} & \multicolumn{2}{|c|}{$\mathrm{C} 3$} & \multicolumn{2}{|c|}{$\mathrm{C} 4$} & \multicolumn{2}{|c|}{ C5 } & \multicolumn{2}{|c|}{ Total } \\
\hline & $\mathrm{n}$ & $\%$ & $\mathrm{n}$ & $\%$ & $\mathrm{n}$ & $\%$ & $\mathrm{n}$ & $\%$ & $\mathrm{n}$ & $\%$ & $\mathrm{n}$ & $\%$ & $\mathrm{n}$ & $\%$ & $\mathrm{n}$ & $\%$ \\
\hline 1983 a 1989 & 47 & 15 & 95 & 30 & 33 & 10 & 26 & 8 & 30 & 9 & 13 & 4 & 75 & 24 & 319 & 100 \\
\hline 1990 a 1999 & 0 & 0 & 10 & 5 & 1 & 1 & 3 & 2 & 2 & 1 & 4 & 2 & 182 & 90 & 202 & 100 \\
\hline 2000 a 2007 & 0 & 0 & 1 & 1 & 1 & 1 & 0 & 0 & 1 & 1 & 1 & 1 & 103 & 96 & 107 & 100 \\
\hline Total & 47 & 8 & 106 & 17 & 35 & 6 & 29 & 5 & 33 & 5 & 18 & 3 & 360 & 57 & 628 & \\
\hline
\end{tabular}

$\mathrm{A}=$ grupo de mulheres com menstruação regular; $\mathrm{B}=$ grupo de mulheres com menstruação irregular; $\mathrm{C} 1=$ grupo de mulheres com menopausa há 1 ano; $\mathrm{C} 2=$ grupo de mulheres com menopausa há 2 ano; $\mathrm{C} 3=$ grupo de mulheres com menopausa há 3 ano; $\mathrm{C} 4=$ grupo de mulheres com menopausa há 4 ano; C5= grupo de mulheres com menopausa há 5 ano.

Tabela 2

Valor mínimo, máximo e mediana da idade e IMC, segundo grupo biológico

\begin{tabular}{|c|c|c|c|c|c|c|c|}
\hline \multirow[b]{2}{*}{ Idade (anos) } & \multicolumn{7}{|c|}{ Grupos Biológicos } \\
\hline & A & $\mathrm{B}$ & $\mathrm{Cl}$ & $\mathrm{C} 2$ & $\mathrm{C} 3$ & $\mathrm{C} 4$ & C5 \\
\hline $\mathrm{N}$ & 47 & 106 & 35 & 29 & 33 & 18 & 360 \\
\hline Mediana & 44 & 50 & 51 & 53 & 55 & 52 & 62 \\
\hline Valor mínimo & 29 & 37 & 38 & 39 & 44 & 45 & 42 \\
\hline Valor máximo & 52 & 62 & 57 & 60 & 68 & 60 & 80 \\
\hline \multicolumn{8}{|l|}{$\mathrm{IMC}\left(\mathrm{kg} / \mathrm{m}^{2}\right)$} \\
\hline $\mathrm{N}$ & 40 & 102 & 35 & 28 & 32 & 17 & 229 \\
\hline Mediana & 27 & 27 & 28 & 25 & 27 & 26 & 27 \\
\hline Valor mínimo & 18 & 17 & 17 & 13 & 17 & 21 & 18 \\
\hline Valor máximo & 39 & 38 & 38 & 43 & 48 & 43 & 48 \\
\hline
\end{tabular}

$\mathrm{A}=$ grupo de mulheres com menstruação regular; $\mathrm{B}=$ grupo de mulheres com menstruação irregular; $\mathrm{C} 1=$ grupo de mulheres com menopausa há 1 ano; $\mathrm{C} 2=$ grupo de mulheres com menopausa há 2 ano; $\mathrm{C} 3=$ grupo de mulheres com menopausa há 3 ano; $\mathrm{C} 4=$ grupo de mulheres com menopausa há 4 ano; C5= grupo de mulheres com menopausa há 5 ano.

Tabela 3

Distribuição percentual da frequiência de Subnutrição, Eutrofia, Sobrepeso e obesidade segundo período de estudo

\begin{tabular}{lccccccr}
\hline & \multicolumn{2}{c}{ Subnutrição } & \multicolumn{2}{c}{ Eutrofia } & \multicolumn{2}{c}{ Sobrepeso } & \multicolumn{2}{c}{ Obesidade } \\
\cline { 2 - 7 } Período & $\mathrm{n}$ & $\%$ & $\mathrm{n}$ & $\%$ & $\mathrm{n}$ & $\%$ & $\mathrm{n}$ \\
\hline 1983 a 1989 & 8 & 3 & 112 & 37 & 106 & 35 & 78 \\
1990 a 1999 & 2 & 2 & 30 & 22 & 56 & 42 & 46 \\
2000 a 2007 & 0 & 0 & 13 & 22 & 17 & 29 & 29 \\
\hline
\end{tabular}

Nota: Classificação do IMC segundo WHO (2012). 
A prevalência de hipertensão, dislipidemia e obesidade aumentou ao longo do tempo, sendo a hipertensão a doença que aparece com maior frequiência em todo o período (Figura 2).

\section{Discussão}

O objetivo do presente estudo foi avaliar a evolução de peso e da frequência de doenças crônicas não transmissíveis de pacientes provenientes do Ambulatório Multidisciplinar de Climatério desde a sua fundação. Os resultados evidenciaram que houve uma modificação na população atendida em relação ao período do climatério em que se encontram. Observou-se um aumento do número de mulheres pertencentes ao grupo biológico C5, ou seja, 5 anos ou mais após a menopausa. Este achado pode estar associado ao envelhecimento que vem ocorrendo na população brasileira. Segundo o Instituto Brasileiro de Geografia e Estatística (IBGE), a expectativa de vida para o sexo feminino, no ano de 2000 foi de 70,4 anos e as proje- ções para os anos de 2010 e 2050 são respectivamente de 78 anos e 81,3 anos. ${ }^{14}$

Com relação à frequência de obesidade e das doenças crônicas não transmissíveis (DCNT) o aumento de sua frequência vem ao encontro do que a literatura tem evidenciado. Em um estudo realizado em Santo André, 307 mulheres regularmente atendidas no Ambulatório de Ginecologia e Obstetrícia do Hospital Universitário do ABC Paulista foram estudadas. Como resultado, 33,6\% apresentavam hipertensão arterial, 4,9\% diabetes mellitus tipo II, 5,2\% hipercolesterolemia e $6,8 \%$ doenças cardiovasculares (DCV), sendo que o IMC médio do grupo foi de 27,3 \pm $4,3 \mathrm{~kg} / \mathrm{m}^{2} .{ }^{15}$ Tais resultados apresentaram-se aquém dos encontrados no presente estudo, que evidenciou uma frequência maior de todas as doenças de uma forma geral.

A presença de comorbidades associadas ao excesso de peso no climatério foi encontrada também em outros estudos. No estudo de De Lorenzi et al. $(2006)^{16}$, foi constatada a prevalência de $49,5 \%$ de

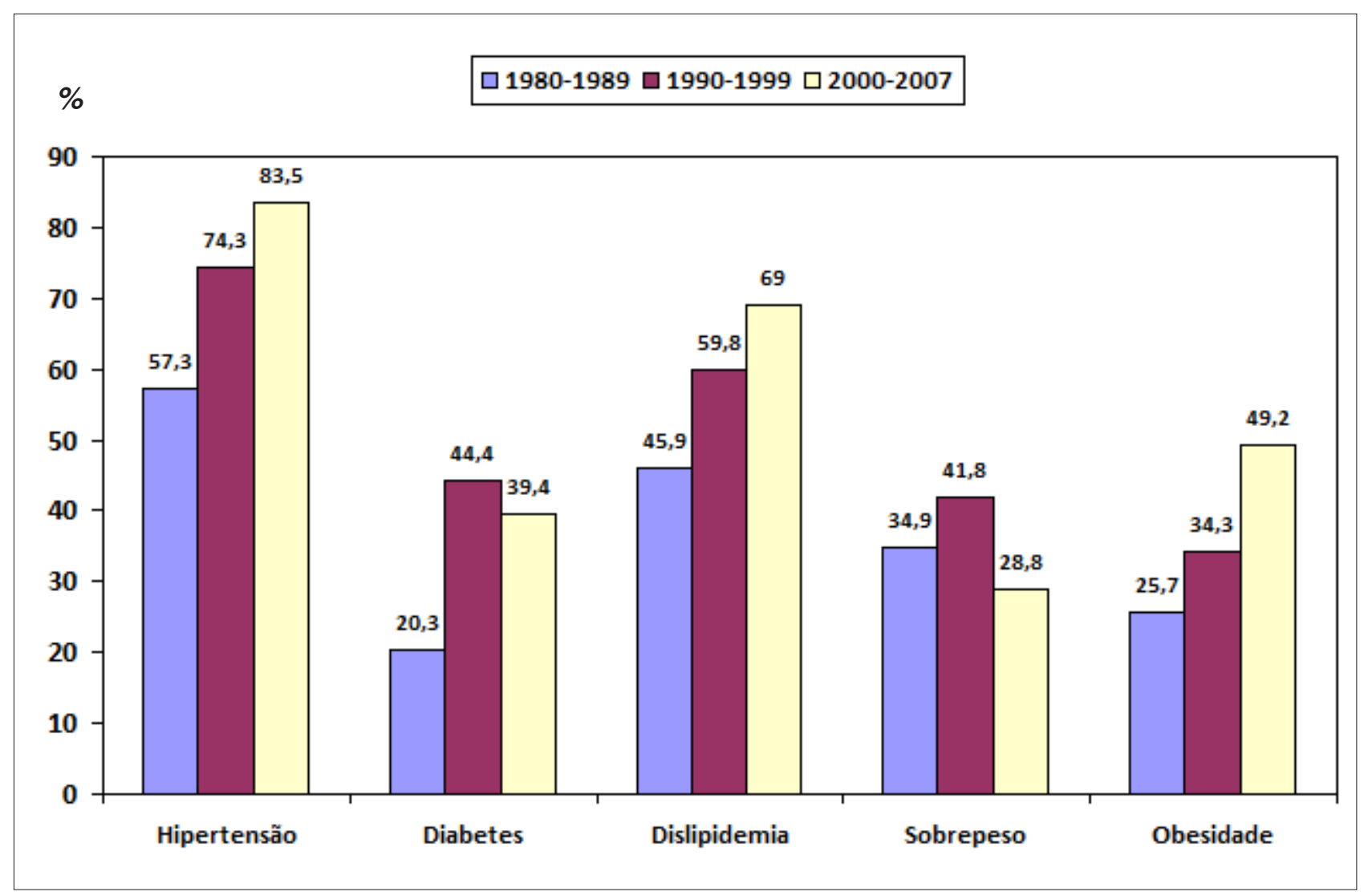

Figura 2: Distribuição percentual da frequência de hipertensão, diabetes, dislipidemia, sobrepeso e obesidade segundo o período de estudo. 
hipertensão e 17,3\% de diabetes tipo II na população estudada (323 mulheres pós-menopausadas). Segundo Genazzani e Gambaciani (2000) ${ }^{17}$, o hipoestrogenismo por muitas vezes vem acompanhado do aparecimento de patologias como cânceres, distúrbios do sono, osteoporose e doenças cardiovasculares (DCV). Para Palasuwan et al. (2011) $)^{18}$, a associação do hipoestrogenismo com a adoção frequente de escolhas alimentares inadequadas também expõem as mulheres pós-menopausadas às $\mathrm{DCV}$.

O ganho de peso e a redistribuição da gordura corporal, típicos dessa fase da vida da mulher, favorecem o aparecimento da síndrome metabólica. ${ }^{19}$ Este estudo mostrou ainda a prevalência média de $60 \%$ de DCV em populações pós-menopausadas contra 13,8\% em pré-menopausadas. No estudo de Williams, Young e Brown (2006) ${ }^{20}$, o ganho de peso de 2,25 kg em um período de 2 anos em uma população de mais de 800 mulheres pós-menopausadas mostrou que estas apresentaram o pior bem-estar físico, o que concordou com outros estudos.

Além disso, a menopausa parece associar-se com a disfunção endotelial, que é o primeiro passo para o desenvolvimento do processo de aterosclerose. Aumentos significativos dos triglicerídeos, colesterol total e LDL-c ocorrem dentro de três a cinco anos após a menopausa natural, a qual se associa também com a forma corporal andróide assumida pelas mulheres, com depósito de gordura abdominal, o que é relacionado com o aumento do risco de doença arterial coronariana. ${ }^{2}$

As DCNTs constituem sério problema de saúde pública, tanto nos países ricos quanto nos de média e baixa renda. Estimativas da Organização Mundial de Saúde (OMS, 2005) apontam que as DCNT já são responsáveis por $60 \%$ de todas as mortes ocorridas no mundo. No Brasil, as DCNTs respondem por $62,8 \%$ do total das mortes por causa conhecida. Estas estatísticas de mortalidade disponíveis para as capitais dos Estados brasileiros indicam que a proporção de mortes por DCNTs aumentou em mais de três vezes entre as décadas de 30 e $90 .^{21,22}$

Chamamos a atenção também para a alta frequência de excesso de peso neste grupo de pacientes. A obesidade está associada à diminuição do bem-estar psíquico, com dificuldade de integração social, baixa auto-estima e estigmatização social. ${ }^{23,24}$ Além disso, pode ter como efeito adicional a redução da capacidade funcional, o que pode refletir na piora da produtividade profissional. ${ }^{25,26}$ Estudos prévios demons- traram que o aumento do IMC tem efeito negativo em muitos aspectos da qualidade de vida. ${ }^{27}$ Associado a isso, as modificações hormonais ocorridas neste período de vida da mulher promovem impactos negativos ainda maiores na qualidade de vida destas mulheres.

Outro fator que pode explicar o presente achado do aumento da frequência observada de excesso de peso ao longo do período é de que o serviço de climatério aqui estudado é um serviço terciário, e a partir de 2000, o serviço passou a priorizar aquelas pacientes que apresentassem comorbidades em estágio mais grave, e, que geralmente, eram acompanhadas por excesso de peso.

O presente estudo apresentou algumas limitações. O fato de ser um estudo observacional não permite a inferência sobre causalidade, ou seja, se o ganho de peso observado levou ao aparecimento das doenças crônicas não transmissíveis, assim como associações entre o envelhecimento, o surgimento das doenças e o ganho de peso. O objetivo do estudo foi analisar as informações contidas nos prontuários médicos, não havendo coleta de dados primários tais como aferições antropométricas e avaliação dietética, o que poderia ter enriquecido a pesquisa. Além disso, o número de mulheres que seguiram com o atendimento no Ambulatório durante todo o período (1983 até 2007) é limitado.

Finalizando, o presente estudo atenta ao fato de que o climatério é uma fase da vida feminina que exige cuidados clínicos e nutricionais. Os profissionais que atendem este grupo de pacientes devem ficar atentos à evolução de peso e à pesquisa diagnóstica de doenças crônicas não-transmissíveis.

\section{Conclusão}

Mulheres climatéricas atendidas em um hospital de nível de atendimento terciário apresentaram um aumento do IMC e da prevalência de doenças crônicas não transmissíveis com o passar do tempo, o que faz urgir um olhar mais atento dos profissionais de saúde a esse grupo populacional.

\section{Agradecimentos}

Agradecemos à colaboração do Serviço de Arquivos Médicos do Hospital das Clínicas de Ribeirão Preto - HCFMRP/USP e ao apoio do GECLINUSP (Grupo de Estudos de Climatério e NutriçãoUSP). 


\begin{abstract}
Study design: Retrospective analysis of medical records.

Purpose: This study aimed to determine the prevalence of obesity, diabetes mellitus, hypertension and dyslipidemia in a group of climacteric women.

Methods: Study of the first patients treated at the Menopause Clinic (ACLI), Department of Obstetrics and Gynecology, School of Medicine of Ribeirão Preto (USP), from 1983 to 2007. Data on weight, height, BMI, biological group, diagnosis of hypertension, diabetes and dyslipidemia was collected from February/ 2008 until April/2008.

Results: From 1983 until 2007, 400 patients were followed up, and 272 had their records registered. Of these 272 patients, 628 were selected queries, and therefore, on average, each woman had three returns. Women over the age of 29 and maximum of 80 years and median $\mathrm{BMI}$ above $25 \mathrm{~kg} / \mathrm{m}^{2}$. The prevalence of diabetes, hypertension and dyslipidemia was respectively $32 \%, 68 \%$ and $54 \%$. The prevalence of NCDs and BMI was higher for the later groups.

Conclusion: Climacteric women treated at a hospital level care center showed a worsening of the BMI and the prevalence of noncommunicable chronic diseases over time, which is urging a closer look at health professionals in this population group.
\end{abstract}

Keywords: Climacteric. Menopause. Hypertension. Diabetes Mellitus. Dyslipidemias. Obesity.

\section{Referências bibliográficas}

1. [NAMS] North American Menopause Society. Clinical challenges of perimenopause: consensus opinion of the NAMS. Menopause. 2000; 7: 5-13.

2. Suen VMM, Bombig GT, Rosa FT, Monteiro TH, Santos RDS, Marchini JS et al. Avaliação clínica retrospectiva de mulheres no período do climatério: A importância da prevenção. Femina. 2006, 34: 607-11.

3. Santos RDS, Suen VMM, lannetta O, Marchini JS. Climacteric, physically active women ingesting their routine diet oxidize more carbohydrates than lipids. Climateric. 2008; 11: $54-60$.

4. Kimura, M; Irahara, M.; Yasui, T.; Saito, S.; Tezuka, M.; Yamano, $\mathrm{S}$. et al. The obesity in bilateral ovariectomized rats is related to a decrease in the expression of leptin receptors in the brain. Biochem Biophys Res Commun. 2002; 290: 134953.

5. Casiglia E, Tikhonoff V, Caffi S, Bascelli A, Schiavon L, Guidotti $F$ et al. Menopause does not affect blood pressure and risk profile, and menopausal women do not become similar to men. J Hipertens. 2008; 26: 1983-92.

6. Cifkova R, Pitha J, Lejskova M, Lanska V, Zecova S. Blood pressure around the menopause: a population study. J Hipertens. 2008; 26: 1946-82.

7. Lovejoy JC. The influence of sex hormones and obesity across female life span. J Women's Health. 2001; 7: 124756.

8. Gambacciani M, Ciaponi M, Cappagli B, De Simone L, Orlandi R, Genazzani AR. Prospective evaluation of body weight and body fat distribution in early port-menopausal women with and without hormonal replacement therapy. Maturitas. 2001; 39: 125-32.

9. Milewicz A, Tworowska U, Demissie M. Menopausal obesity - myth or fact? Climacteric. 2001; 4: 273-83.

10. Monteiro RCA, Riether PTA, Burini RC. Efeito de um programa misto de intervenção nutricional e exercício físico sobre a composição corporal e os hábitos alimentares de mulheres obesas em climatério. Rev Nutr. 2004; 17: 479-89.
11. Eshtiaghi R, Esteghamati A, Nakhjavani M. Menopause is an independent predictor of metabolic syndrome in Iranian women. Maturitas. 2010; 65: 262-6.

12. Binfa L, Blümel M. Obesidad, estrógenos y salud de la mujer. Rev Chil Obstet Ginecol. 2001; 66: 340-6.

13. World Health Organization. BMI Classification. [Acess in July 1st, 2012]. Available in <http://apps.who.int/bmi/>

14. [IBGE] Instituto Brasileiro de Geografia e Estatística. Disponível em: <http://www.ibge.gov.br/home/estatistica/populacao/ projecao_da_populacao/2008/projecao.pdf>. Acesso em: 25 de janeiro de 2010.

15. Ferreira JA, Pompei LM, Fernandes CE, Azevedo LH, Peixoto $\mathrm{S}$. Breast arterial calcification is a predictive factor of cardiovascular disease in Brazilian postmenopausal women. Climacteric 2009; 28: 1-6.

16. De Lorenzi DRS, Bacarat EC, Saciloto B, Padilha Jr I. Fatores associados à qualidade de vida após a menopausa. Rev Assoc Méd Bras. 2006; 52: 312-17.

17. Genazzani AR, Gambacciani M. Controversial issues in climacteric medicine: cardiovasvular disease and hormonal replacement therapy. Climacteric. 2000; 3: 233-40.

18. Palasuwan A, Margaritis I, Soogarun S, Rousseau AS. Dietary intakes and antioxidant stauts in mind-body exercising pre- and postmenopausal women. J Nutr Health Aging. 2011; 15: $577-84$.

19. Petri-Nahas EA, Padoani NP, Nahas-Neto J, Orsatti FL, Tardivo AP, Dias R. Metabolic syndrome and its associated risk factors in Brazilian postmenopausal women. Climacteric. 2009; 12: $431-8$.

20. Williams LT, Young AF, Brown WJ. Weight gained in two years by a population of mid-aged women: how much is too much? Int J Obes. 2006; 30: 1229-33.

21. Brasil. Ministério da Saúde. Secretaria de Vigilância em Saúde. Secretaria de Gestão Estratégica e Participativa. Vigitel Brasil 2008: vigilância de fatores de risco e proteção para doenças crônicas por inquérito telefônico/Ministério da Saúde, Secretaria de Vigilância em Saúde, Secretaria de Gestão Estratégica e Participativa-Brasília: Ministério da Saúde, 2009. 112 p. 
22. Moura EC, Neto OLM, Malta DC, Moura L, Silva NN, Bernal R et al. Vigilância de fatores de risco para doenças crônicas por inquérito telefônico nas capitais dos 26 estados brasileiros e no Distrito Federal (2006). Rev Bras Epidemiol 2008; 11(supl 1): 20-37.

23. Orsi JVA, Nahas FX, Gomes HC, Andrade CHV, Veiga DF, Novo NF et al. Impacto da obesidade na capacidade funcional de mulheres. Rev Assoc Med Bras 2008; 54: 106-9.

24. Rodriguez MCN, Santana PS, Velasco PP, Gonzales NS, Ruiz EM, Medina, RC et al. Estilo de vida, nível socioeconómico y morbilidad en mujeres posmenopáusicas con obesidad de grados II y III. Endocrinol Nutr. 2009; 56: 227-32.
25. Jia H, Lubtikin El. The impact of obesity on health-related quality-of-life in the general adult US population. J Public Health 2005; 27: 156-64.

26. Martins MAD, Nahas EAP, Nahas-Neto J, Uemura G, Buttros DAB, Traiman P. Qualidade de vida em mulheres na pósmenopausa, usuárias e não usuárias de terapia hormonal. Rev Bras Ginecol Obstet. 2009; 31:196-202.

27. Ul-Haq Z, Mackay DF, Fenwick E, Pell JP. Impact of metabolic comorbidity on the association between body mass index and health-related quality of life: a Scotland-wide crosssectional study of 5,608 participants. BMC Public Health 2012 $24: 143$ 